\title{
A SIMPLE EXPRESSION FOR THE CASIMIR OPERATOR ON A LIE GROUP
}

\author{
MARY F. ANDERSON ${ }^{1}$
}

\begin{abstract}
The expression for the Casimir operator for a real semisimple Lie group $G$ in terms of coordinates given by the Iwasawa decomposition $G=K A N$ reduces on $G / N$ to the difference of an elliptic operator with constant coefficients on $A$ and an invariant operator on $M$. This result immediately identifies the principal series of induced representations with representations defined on the eigenspaces of the restriction of the Casimir operator to $G / N$.
\end{abstract}

When the Casimir operator $\Gamma$ for a real semisimple Lie group $G$ is expressed as a differential operator on the group in coordinates given by the Iwasawa decomposition $G=K A N$, it is seen, when restricted to $G / N$, to be the difference of an elliptic operator with constant coefficients on $A$ and the Casimir operator on $M$. This result immediately identifies the principal series of induced representations with representations which arise from the restriction of the left regular representation of $G$ to certain eigenspaces of $\Gamma_{G / N}$. On the other hand, when this expression of $\Gamma$ is restricted to $K \backslash G$, it is seen to be a second order invariant operator on $A N$. This allows us to identify the symmetric space $G / K$ with the solvable group $A N$.

To the best of my knowledge, this result has not appeared in the literature, even though it is intrinsic to Harish-Chandra's work and is suggested by his expression for the Haar measure in terms of the Iwasawa decomposition. The radial part of the invariant operators on $G$ has been computed by Vilenkin, and that of the operators on the Lie algebra $g$ by Varadarajan and his students. Enright and Varadarajan use an expression of $\Gamma$ by Hotta and Parasarathy to construct a model of the discrete series on certain eigenspaces of $\Gamma$. However, their expression, which stems from a root space decomposition of $\mathfrak{g}$ with respect to root vectors for a compact Cartan subalgebra, is algebraic in nature and valid only for those groups with discrete series. Ehrenpreis constructed models of the principal series and the discrete series on spaces of eigenfunctions of $\Gamma$. However, until present, his work has been restricted to $G / K$ and $G / M N$.

Notation. For $g, h \in G$ and $X \in \mathfrak{g}$, let $g X h$ represent the linear functional

Received by the editors May 30, 1978.

AMS (MOS) subject classifications (1970). Primary 22E30; Secondary 22 E60.

'I am grateful to the Department of Mathematics at the University of California, Berkeley for hospitality extended to me during the preparation of this paper.

(C) 1979 American Mathematical Society 0002-9939/79/0000-0572/\$02.50 
on $C^{1}(G)$ defined by

$$
g X h \cdot f=\left.\frac{d}{d t} f(g(\exp t X) h)\right|_{t=0} .
$$

If $G$ has a subgroup decomposition $G=H_{1} H_{2}$, where $H_{1}$ and $H_{2}$ represent closed subgroups of $G$, and $\mathfrak{g}=\mathfrak{h}_{1} \oplus \mathfrak{h}_{2}$ represents a decomposition of the Lie algebra of $G$ into a sum of the Lie algebras of $\mathfrak{h}_{1}$ and $\mathfrak{h}_{2}$, this notation facilitates the expression of the left and right invariant differential operators on $G$ in terms of left and right invariant differential operators on the subgroups. To see this, assume $g=h_{1} h_{2}$; then

$$
X g=X h_{1} h_{2}=h_{1} \operatorname{ad}\left(h_{1}^{-1}\right) X h_{2} .
$$

Assume now that $\operatorname{ad}\left(h_{1}^{-1}\right) X=\alpha H_{1}+\beta H_{2}$ for some $H_{1} \in \mathfrak{h}_{1}$ and some $H_{2} \in$ $\mathfrak{h}_{2}$. Then

$$
X g=\alpha h_{1} H_{1} h_{2}+\beta h_{1} H_{2} h_{2},
$$

and we see the right invariant operator obtained from $X$ may be expressed as a sum of a differential operator on $H_{1}$ and a right invariant operator on $H_{2}$.

To use this notion to obtain an expresssion for the Casimir operator in terms of operators on teh subgroups $K, A$, and $N$ as they appear in the Iwasawa decomposition $G=K A N$, we must choose a convenient basis for $g$ and find expressions for the adjoint actions of elements of $t$ and $a$ on these basis vectors. To this end, let $\mathfrak{h}_{c}$ be a Cartan subalgebra of $\mathfrak{g}_{c}$ and let $\mathfrak{g}$ have the Cartan decomposition $\mathfrak{g}=\mathfrak{f}+\mathfrak{p}$. As usual, let $\Delta_{+}=\left\{\alpha_{1}, \ldots, \alpha_{n}\right\}$ be the set of roots which are strictly positive on $a$ with respect to some order, and assume the roots $\alpha_{1}, \ldots, \alpha_{r}$ are simple, where $r$ is the rank of $G$. Let $N_{\alpha} \in \mathfrak{n}$ be the standard root vector for the root $\alpha$ and define $P_{\alpha} \in \mathfrak{p}$ and $K_{\alpha} \in \mathfrak{f}$ by

$$
\sqrt{2} P_{\alpha}=N_{\alpha}+N_{-\alpha}, \quad \sqrt{2} K_{\alpha}=N_{\alpha}-N_{-\alpha} \text {. }
$$

For the simple roots $\alpha_{i}, i=1, \ldots, r$, let $H_{\alpha_{i}}$ be the normalized element of a satisfying

$$
\alpha_{i}(H)=\left\langle H_{\alpha_{i}}, H\right\rangle .
$$

A convenient orthonormal basis for $\mathfrak{p}$ is provided by the set of vectors

$$
X_{i}= \begin{cases}P_{\alpha_{i}}, & \text { if } i=1, \ldots, n, \\ H_{\alpha_{i-n}}, & \text { if } i=n+1, \ldots, n+r .\end{cases}
$$

Similarly, the vectors $K_{\alpha_{i}}$, a complete set of root vectors for $\mathfrak{m} \cap \mathfrak{h}_{c}$ and an orthonormal basis for $\mathfrak{m} \cap \mathfrak{h}_{c}$, form a convenient orthonormal basis for $\mathfrak{f}$.

The vectors $K_{\alpha_{i}}, N_{\alpha_{i}}$, and $H_{\alpha_{i}}$ give the following operators of differentiation with respect to the Iwasawa coordinates:

$$
\begin{aligned}
& D_{K_{\alpha_{i}}}^{L} f(g)=D_{K_{\alpha_{i}}}^{L} f(k a n)=k K_{\alpha_{i}} \text { an } \circ f, \\
& D_{N_{\alpha_{i}}}^{R} f(g)=D_{N_{\alpha_{i}}}^{R} f(k a n)=k a N_{\alpha_{i}} n \circ f, \\
& D_{H_{\alpha_{i}}} f(g)=D_{H_{\alpha_{i}}} f(k a n)=k H_{\alpha_{i}} \text { an } \circ f .
\end{aligned}
$$


$D_{H_{\alpha_{i}}}$ is easily represented as a partial derivative. Let $s \in R^{r}$ and represent $a \stackrel{a_{i}}{\epsilon} A$ by $a=a(\mathbf{s})=\exp \left(\sum_{i=1}^{r} s_{i} H_{\alpha_{i}}\right)$. Then $D_{H_{a_{i}}}=\partial / \partial s_{i}$.

We now prove a lemma needed to express the Casimir operator in terms of the above differential operators.

LEMMA 1. Let $\left(k_{i j}\right)$ be the matrix expression of $\operatorname{ad}(k)$ on $\mathfrak{p}$ in terms of the basis vectors $X_{j}, j=1, \ldots, n+r$. Let $1 \leqslant i \leqslant n$. Then

$$
\sum_{j=1}^{n+r} k_{j i}\left(D_{K_{\alpha_{i}}}^{L} k_{j i^{\prime}}\right)=\left\{\begin{array}{l}
0, \quad \text { if } i^{\prime} \leqslant n, \\
\alpha_{i}\left(H_{\alpha_{i^{\prime}-n}}\right), \text { if } n+1<i \leqslant n+r .
\end{array}\right.
$$

Let $\rho=\frac{1}{2} \sum_{i=1}^{n} \alpha_{i}$. Then

$$
2 \rho\left(H_{\alpha_{i^{\prime}-n}}\right)=\sum_{i=1}^{n} \alpha_{i}\left(H_{\alpha_{i^{\prime}-n}}\right)
$$

Proof.

$$
\begin{aligned}
& k_{j i^{\prime}}=\left\langle\operatorname{ad}(k) X_{i^{\prime}}, X_{j}\right\rangle \text {, } \\
& D_{K_{\alpha_{i}}}^{L} k_{j i^{\prime}}=\left.\frac{d}{d t}\left\langle\operatorname{ad}\left(k \exp t K_{\alpha_{i}}\right) X_{i^{\prime}}, X_{j}\right\rangle\right|_{t=0}, \\
& \left.\frac{d}{d t} \operatorname{ad}\left(\exp t K_{\alpha_{i}}\right) X_{i^{\prime}}\right|_{t=0}=\left[K_{\alpha_{i}}, X_{i^{\prime}}\right]
\end{aligned}
$$

Thus, if $X_{i^{\prime}}=P_{\alpha_{i}}$, and $i \neq i^{\prime}$,

$$
\left[K_{\alpha_{i}}, X_{i^{\prime}}\right]=\left[K_{\alpha_{i}}, P_{\alpha_{i^{\prime}}}\right]=d\left(\alpha_{i}+\alpha_{i^{\prime}}\right) P_{\alpha_{i}+\alpha_{i^{\prime}}}-d\left(\alpha_{i}-\alpha_{i^{\prime}}\right) P_{\alpha_{i}-\alpha_{i^{\prime}}}
$$

where $d$ is a function defined on $\mathfrak{h}_{c}^{*}$ by

$$
d(\beta)= \begin{cases}1, & \text { if } \beta \text { is a root of } \mathfrak{h}_{c}, \\ 0, & \text { if } \beta \text { is not a root of } \mathfrak{h}_{c} .\end{cases}
$$

If $i=i^{\prime}$,

$$
\left[K_{\alpha_{i}}, P_{\alpha_{i}}\right]=H, \text { for some } H \in \mathfrak{a}
$$

If $X_{i^{\prime}}=H_{\alpha^{\prime}-n}$,

$$
\left[K_{\alpha_{i}}, X_{i^{\prime}}\right]=\left[K_{\alpha_{i}}, H_{\alpha_{i^{\prime}-n}}\right]=\alpha_{i}\left(H_{\alpha_{i^{\prime}-n}}\right) P_{\alpha_{i}}
$$

Thus, if $j^{\prime} \leqslant n$,

$$
D_{K_{\alpha_{i}}}^{L} k_{j i^{\prime}}=\sum_{\mu=1}^{n+r} \gamma_{j \mu} k_{j \mu}
$$

for constants $\gamma_{j \mu}$ of which $\gamma_{j i}=0$. If $i^{\prime}>n$,

$$
D_{K_{\alpha_{i}}}^{L} k_{j i^{\prime}}=\alpha_{i}\left(H_{\alpha_{i^{\prime}-n}}\right) k_{j i} \text {. }
$$

(9) now follows from the orthogonality of $\operatorname{ad}(k)$ on $\mathfrak{p}$.

We are now able to prove the desired result.

THEOREM 1. Let $\Gamma$ be the Casimir operator on $G$ and $\Gamma_{M}$ the Casimir operator on $M$. Then the expression of $\Gamma$ as a differential operator in the Iwasawa 
coordinates $g=k a(\mathbf{s}) n$ is

$$
\begin{aligned}
\Gamma= & \sum_{i=1}^{r}\left(\frac{\partial^{2}}{\partial s_{i}^{2}}+2 \rho\left(H_{\alpha_{i}}\right) \frac{\partial}{\partial s_{i}}\right)-\Gamma_{M} \\
& +4 \sum_{i=1}^{n}\left(e^{-2 \alpha_{i}(s)}\left(D_{N_{\alpha_{i}}}^{R}\right)^{2}-e^{-\alpha_{i}(\mathrm{~s})} D_{K_{\alpha_{i}}}^{L} D_{N_{\alpha_{i}}}^{R}\right) .
\end{aligned}
$$

Proof. Recall that

$$
\Gamma=\sum_{j=1}^{n+r}\left(X_{j} g\right)^{2}-\sum_{j=1}^{n}\left(K_{\alpha, g} g\right)^{2}-\Gamma_{M}
$$

First, we find an expression for $X_{j} g$ in terms of $D_{K_{\alpha_{i}}}^{L}, D_{N_{a_{i}}^{R}}^{R}$, and $D_{H_{a_{i}}}$.

$$
X_{j} g=X_{j} k a n=k \operatorname{ad}\left(k^{-1}\right) a n=k \sum_{i=1}^{n+r} k_{i j} X_{i} a n .
$$

Consider the terms in the last summation in (21). If $i \leqslant n$, then $X_{i}=P_{\alpha_{i}}$ and

$$
\begin{aligned}
k X_{i} a n & =k P_{\alpha_{i}} \text { an }=k\left(-K_{\alpha_{i}}+2 N_{\alpha_{i}}\right) a n \\
& =-k K_{\alpha_{i}} \text { an }+e^{-\alpha_{i}(\mathbf{s})} k a N_{\alpha_{i}} n=-D_{K_{\alpha_{i}}}^{L}+2 D_{N_{\alpha_{i}}}^{R} .
\end{aligned}
$$

On the other hand, if $i>n$, then $X_{i}=H_{\alpha_{i-n}}$, and

$$
k X_{i} a n=\frac{\partial}{\partial s_{i-n}} .
$$

Thus

$$
\left(X_{j} g\right)^{2}=\left(\sum_{i=1}^{n} k_{j i}\left(-D_{K_{\alpha_{i}}}^{L}+2 e^{-\alpha_{i}(\mathrm{~s})} D_{N_{\alpha_{i}}}^{R}\right)+\sum_{i=1}^{r} k_{j i+n} \frac{\partial}{\partial s_{i}}\right)^{2} .
$$

The contribution to the Casimir operator from $\mathfrak{p}$ is simply $\sum_{j=1}^{n+r}\left(X_{j} g\right)^{2}$.

Since $\operatorname{ad}(k)$ is an orthogonal transformation on $\mathfrak{p}$,

$$
\sum_{j=1}^{n+r} k_{j i} k_{j \mu}= \begin{cases}0, & \text { if } i \neq \mu \\ 1, & \text { if } i=\mu .\end{cases}
$$

Thus, when the RHS of (24) is summed over $j,(25)$ and the result (9) of Lemma 1 show that

$$
\sum_{j=1}^{n+r}\left(X_{j} g\right)^{2}=\sum_{j=1}^{n}\left(-D_{K_{\alpha_{j}}}^{L}+2 e^{-\alpha_{j}(s)} D_{N_{\alpha_{j}}}^{R}\right)^{2}+\sum_{i=1}^{r}\left(\frac{\partial^{2}}{\partial s_{i}^{2}}+2 \rho\left(H_{\alpha_{i}}\right) \frac{\partial}{\partial s_{i}}\right) .
$$

We now combine (26) and (20) to obtain (19).

The restriction of $\Gamma$ to functions invariant under right multiplication by $N$ has particularly the simple form

$$
\Gamma_{G / N}=\sum_{i=1}^{r}\left(\frac{\partial^{2}}{\partial s_{i}^{2}}+2 \rho\left(H_{\alpha_{i}}\right) \frac{\partial}{\partial s_{i}}\right)-\Gamma_{M} .
$$

This immediately results in the identification of the pricipal series with representations on certain eigenspaces of $\Gamma_{G / N}$. 
Corollary. Let $\sigma \in R^{r}$ represent an element of $a_{c}$ and let $\pi_{\mathrm{m}}$ be an irreducible representation of $M$ with character $\chi_{\mathrm{m}}$. Then the restrictions of the following three representations of $G$ to their respective $K$-finite analytic subspaces are equivalent:

(i) the representation $g \rightarrow V(g ; \sigma, \mathbf{m})$ induced by the representation

$$
m a(\mathbf{s}) n \rightarrow \pi_{\mathbf{m}}(m) \exp (\sigma, \mathbf{s})
$$

of $M A N$;

(ii) the multiplier representation $g \rightarrow \mathcal{U}(g ; \sigma, \mathrm{m})$ on the $\pi_{\mathrm{m}}$ right invariant subspace $L_{\mathrm{m}}^{2}(K)$ of $L^{2}(K)$ defined by

$$
\mathcal{Q}(g ; \boldsymbol{\sigma}, \mathbf{m}) f(k)=\exp \left(\boldsymbol{\sigma}-\rho, \mathbf{s}_{g}\right) f\left(k_{g}\right),
$$

where, when $k$ is taken to be an element of $G$,

$$
g^{-1} k=k_{g} a\left(s_{g}\right) n_{g} ; \text { and }
$$

(iii) the restriction of the left regular representation of $G$ to the subspaces of analytic functions on $G / N$ which satisfy

$$
\begin{gathered}
\Gamma_{G / N} f=\left\{(\boldsymbol{\sigma}, \boldsymbol{\sigma})-\gamma_{M}(\mathbf{m})\right\} f, \\
\left.D_{H_{\alpha_{j}}} f\right|_{\mathbf{s}=0}=\left.\frac{\partial}{\partial s_{j}} f(k a(\mathbf{s}))\right|_{\mathbf{s}=0}=\left.\left(\sigma_{j}-\rho\left(H_{\alpha_{j}}\right)\right) f(k a(\mathbf{s}))\right|_{\mathbf{s}=0},
\end{gathered}
$$

and

$$
\int_{M} f(x m) \bar{\chi}_{\mathbf{m}} d m=f(x)
$$

where $\bar{\chi}_{\mathbf{m}}$ represents the normalized character of $\pi_{\mathbf{m}}$.

Proof. The equivalence of (i) and (ii) follows easily from the identification of $K$ and $G / A N$. Assume $\pi_{\mathrm{m}}$ acts on a Hilbert space $H_{\mathrm{m}}$ which has an inner product $(,)_{\mathrm{m}}$ and a basis $B_{\mathrm{m}}$. Let $L_{\mathrm{m}}(G)$ represent the set of all $H_{\mathrm{m}}$ valued functions on $G$ which satisfy

$$
f(g m a(\mathbf{s}) n)=\pi_{\mathrm{m}}(m) \exp (\sigma-\rho, \mathbf{s}) f(g) .
$$

Then, if $g$ is expressed in its Iwasawa coordinates $g=k a n, f$ is seen to be completely determined by its values on $K$. Furthermore, each $v \in B_{\mathbf{m}}$ determines an intertwining operator $T_{v}$ of the representations $V(g ; \boldsymbol{\sigma}, \mathbf{m})$ and $\mathcal{Q}(g ; \boldsymbol{\sigma}, \mathbf{m})$, where $T_{v}$ is defined by

$$
T_{v} f(g)=T_{v} f(k a n)=(f(k), v)_{\mathbf{m}}
$$

A straightforward application of the Peter-Weyl theorem shows the maps are injective, and that $L_{\mathrm{m}}^{2}(K)$ is spanned by the subspaces $T_{v} L_{\mathrm{m}}(G)$ as $v$ runs through all of $B_{\mathbf{m}}$.

The equivalence of (ii) and (iii) follows naturally from Theorem 1. If $f \in L_{m}^{2}(K)$ is analytic, then the function $f(x)$ defined on $G / N$ by

$$
f(x)=f(k a(\mathbf{s}))=\exp (\sigma-\rho, \mathbf{s}) f(k)
$$


satisfies (31)-(33). It should be noted that, for fixed $\sigma$, the energy integral for the Casimir operator for a function $f$ with Cauchy data (32) is

$$
\int_{K}\left\{|f(k)|^{2}+\sum_{i=1}^{r}\left|\frac{\partial}{\partial s_{i}} f(k a(\mathbf{s}))\right|_{s=0}^{2}\right\} d k=(1+|(\sigma-\rho, \sigma-\rho)|) \int_{K}|f(k)|^{2} d k .
$$

Thus, the eigenspace representation described in (iii) becomes a Hilbert space representation with the inner product defined by the energy integral (37). The uniqueness of solutions to (31) with Cauchy data (32) and (33) proves the $K$-finite equivalence of the representations of (ii) and (iii).

COROLlaRY. The symmetric space $K \backslash G$ may be identified in a natural way with the solvable group $A N$. In this identification, the Laplacian is

$$
\Delta=\sum_{i=1}^{r}\left(\frac{\partial^{2}}{\partial s_{i}^{2}}+2\left(H_{\alpha_{i}}\right) \frac{\partial}{\partial s_{i}}\right)+4 \sum_{j=1}^{n} e^{-2 \alpha_{i}(s)}\left(D_{N_{\alpha_{i}}}^{R}\right)^{2}
$$

\section{REFERENCES}

1. R. Courant and D. Hilbert, Methods of mathematical physics, vol. 2, Interscience, New York, 1962.

2. L. Ehrenpreis, The use of partial differential equations for the study of group representations, Proc. Sympos. Pure Math., vol. 26, Amer. Math. Soc., Providence, R. I., 1973, pp. 317-320.

3. T. J. Enright and V. S. Varadarajan, On an infinitesimal characterisation of the discrete series, Ann. of Math. 102 (1975), 1-15.

4. Harish-Chandra, Harmonic analysis on reductive groups. I, J. Functional Analysis 19 (1975), 104-204.

5. __, Harmonic analysis on reductive groups. II, Invent. Math. 36 (1976), 1-55.

6. Harmonic analysis on reductive groups. III, Ann. of Math. (2) 104 (1976), 117-201.

7. S. Helgason, Differential geometry and symmetric spaces, Academic Press, New York, 1962.

8. R. Parasarathy, Dirac operator and the discrete series, Ann. of Math. 96 (1972), 1-30.

9. P. C. Trombi and V. S. Varadarajan, Spherical transforms on semisimple Lie groups, Ann. of Math. 94 (1971), 246-303.

10. M. Pazirandeh, Invariant differential operators on a real semisimple Lie algebra and their radial components, Trans. Amer. Math. Soc. 182 (1973), 119-131.

Department of Mathematics, University of California, Berkeley, California 94720 